Final draft of (2005) paper published in B. E. Rogowitz, T. N. Pappas, S. J. Daly (Eds.) "Human Vision and Electronic Imaging X", proceedings of SPIE-IS\&T Electronic Imaging, SPIE Vol 5666 (pp. 503-514).

\title{
The perception of linear self-motion
}

\author{
Frank H. Durgin ${ }^{*}$, Laura F. Fox ${ }^{\text {ab }}$, Evan Schaffer ${ }^{\mathrm{a}}$, Rabi Whitaker ${ }^{\mathrm{a}}$ \\ ${ }^{a}$ Dept. of Psychology, Swarthmore College, 500 College Ave., Swarthmore, PA 19081; \\ ${ }^{b}$ Dept. of Brain and Cognitive Sciences, MIT, 77 Massachusetts Ave., Cambridge, MA 02139;
}

\begin{abstract}
VR lends itself to the study of intersensory calibration in self-motion perception. However, proper calibration of visual and locomotor self-motion in VR is made complicated by the compression of perceived distance and by unfamiliar modes of locomotion. Although adaptation is fairly rapid with exposure to novel sensorimotor correlations, here it is shown that good initial calibration is found when both (1) the virtual environment is richly structured in near space and (2) locomotion is on solid ground. Previously it had been observed that correct visual speeds seem too slow when walking on a treadmill. Several principles may be involved, including inhibitory sensory prediction, distance compression, and missing peripheral flow in the reduced FOV. However, though a richly-structured near-space environment provides higher rates of peripheral flow, its presence does not improve calibration when walking on a treadmill. Conversely, walking on solid ground still shows relatively poor calibration in an empty (though welltextured) virtual hallway. Because walking on solid ground incorporates well-calibrated mechanisms that can assess speed of self-motion independent of vision, these observations suggest that near space may have been better calibrated in the HMD. Near-space obstacle avoidance systems may also be involved. Order effects in the data from the treadmill experiment indicate that recalibration of self-motion perception occurred during the experiment.
\end{abstract}

Keywords: Self-motion, virtual reality, calibration, locomotion, immersion, optic flow, vestibular

\section{INTRODUCTION}

There are many sources of information for the perception of self-motion. Among these are optic flow, efference copy, kinesthesis, and vestibular signals. Although recovering speed information from optic flow is complicated because retinal velocities depend on distance as well as speed, the use of ground-plane flow, which typically maintains a fixed distance at any given angle of regard, may be directly useful for estimating self-motion. ${ }^{1}$ A number of researchers have shown that optic flow can be used to estimate distance of self-motion. ${ }^{2-4}$ However, in many settings it proves to be unnecessary (e.g., visually-directed walking and walking in a crowd), and in others it proves misleading (e.g., the visual sense of speed in a high SUV vs. a low sports car.) Motor control parameters and kinesthesis are probably fairly important to estimating the speed of self-motion in the absence of vision, but these need to be scaled by experience. Indeed, when walking without visual feedback, calibration of self-motion seems to break down when people are required to walk more rapidly or more slowly than they would normally. ${ }^{5}$ Although inertial (e.g., vestibular) estimates of self-motion are sometimes treated as informative, ${ }^{6,7}$ these seem to be quite limited with respect to the perception of speed and distance. ${ }^{5,8}$ Nonetheless, all these kinds of information (along with other information) normally co-occur during locomotion, and it seems likely that inter-sensory interactions are the rule. ${ }^{9-12}$ The intercalibration of various sources of information produces integrated perceptual expertise in normal self-motion perception that may have a common spatial sense at its core and that cannot be easily analyzed into separate components. Nonetheless, in the present experiments we ask people to evaluate the relationship between visual and non-visual estimates of self-motion in a virtual environment. Our results suggest that people are able to detect discrepancies in conditions that resemble normal walking among near objects, but provide evidence of failures of treadmill locomotion to mimic normal walking and limitations of objectless optic flow in VR.

Before presenting our experiments, we will review past evidence concerning matches between perceived visual flow and locomotor speed and consider evidence concerning the sources of perceptual information available for making these judgments. We will also review evidence that emphasizes surprising interactions among sources of information relevant

\footnotetext{
*fdurgin1@swarthmore.edu; phone 1610328 8678; fax 1610328 7814; www.swarthmore.edu/SocSci/fdurgin1
} 
to the perception of self-motion and limitations of the various sources of self-motion information when taken separately. Although our ultimate conclusions are primarily practical and methodological, the theoretical underpinnings of the research require careful articulation.

\subsection{Theoretical underpinnings}

We note here that some might argue that there is something of a category error entailed by referring to self-motion "perception." Whereas we regard self-motion the object of a multisensory perceptual system, ${ }^{12}$ Gibson in "The senses considered as perceptual systems", treats self-motion perception as "proprioception" and distinguishes it from perception, which is of the world. ${ }^{13}$ Optic flow information, during locomotion, contains motion parallax information specifying the structure of the world as well as information specifying ones one movement through that world. This leads to an ambiguity. Is the optic flow that specifies self-motion idiothetic? For our purposes the answer is no. Optic flow is exteroceptive (allothetic) information that accompanies self-motion and can be used to estimate self-motion. Our bodies are part of the world. Perception of ones self (and its movement) in the world is properly regarded as perception, even it involves the specialized term of "proprioception" and has access to idiothetic (interoceptive) information as well. Gibson's point was that proprioception is not limited to interoceptive information (internal senses).

Information about self-motion is both integrated and segregated. Visual motion can produce vivid sensation of selfmotion (vection), but can also be observed in a detached manner. Thus, although self-motion perception is multimodal, at least some of the various sources of self-motion information can remain segregated. That is, we can ask observers not only to judge their self-motion, but to compare the information they have from different modalities. Consider the following scenario: I move you passively through the world on a cart while I show you an independently controlled optic flow field in an HMD. I can ask you several things about that situation: How fast were you moving? How fast did the optic flow seem to be? Did the world producing the flow seem to move, or was it stationary as you moved through it? Was the flow too fast or too slow given the speed you actually moved? Which of these questions requires which information?

To answer the first question (How fast you were moving?) asks you to refer to the physical motion. It is likely that visual motion would dominate this perception, however. ${ }^{14}$ To answer the second question (How fast did the optic flow seem to be?) requires that you refer to the visual motion alone, but there is good reason to believe it would be influenced by the rate of physical motion. ${ }^{15-19}$ If I ask you the third question (Did the world seem to move?) I might intend to be asking you the same thing as the fourth question (Was the flow too slow or too fast?), but world stability assumptions could produce a sense that the world did not move while still allowing one to make a comparative judgment between two different sources of information about ones own self-motion. It is this comparison of visual flow to perceived selfmotion that will primarily concern us in the present paper. Such comparisons require "intercalibration" of multiple systems.

In the present studies, rather than passive self-motion, we ask people to engage in active self-motion (walking) or simulated active self motion (treadmill walking) and to evaluate whether the rate of optic flow they receive through an HMD is appropriate to their self-motion. It is not obvious on the face of it whether this is a cognitive task, or a perceptual one. To the extent that people can make clear "visceral" judgments about the relative speed of locomotion and visual flow, we regard the judgment as perceptual. It is important to note that this task does not allow us to know the rate of perceived self-motion the observer is experiencing. Instead, we use it to evaluate the inter-calibration of the relevant sources of self-motion information.

\subsection{Precursor studies of visual comparisons to non-visual self-motion perception}

Pelah and Barlow reported that the rate of optic flow while walking appeared exaggerated following treadmill running. ${ }^{20}$ They interpreted this finding as a kind of motor-contingent visual aftereffect resulting from the novel mapping between vision and action or between vision and the other sensory signals that accompanied action. We call this the "flow" effect to emphasize that it affects apparent optic flow rates. The fact that people can notice that flow is too fast suggests that they can indeed be separately aware of physical self-motion and optic flow.

Consider however, that recent studies of flow-rate perception during biomechanical self-motion suggest that perceived flow rates are normally reduced during self-motion relative to their appearance while stationary. ${ }^{19}{ }^{21-23}$ Durgin, Gigone 
and Scott found that judgments of visual speeds were reduced during walking by about 40 percent of walking speed. ${ }^{19}$ Somewhat smaller subtractions were found during treadmill locomotion and during passive self-motion. They argued that these reductions in perceived speed were most consistent with the kind of inter-channel inhibition proposed by Barlow. ${ }^{24}$ If the perceived rate of flow is normally reduced during self-motion, the flow effect reported by Pelah and Barlow following treadmill adaptation might be viewed as a release from inhibition following a decoupling of motor activity and visual feedback. That is, the perceived flow after adaptation might have been more "accurate" in one sense, though exaggerated relative to that normally experienced during self-motion.

Pelah and Barlow measured the flow effect in a somewhat indirect way. ${ }^{20}$ After running on a treadmill, people were asked to walk a short path at a fairly slow pace (specified temporally) while noting the apparent optic flow. They were then asked to walk the same path repeatedly at a speed that maintained the same apparent optic flow. Over a series of trials, people sped up as they walked, by about $30 \%$, ostensibly in order to maintain the same visual flow as the aftereffect wore off. One drawback of this method was that merely by returning to a normal walking pace over time (as a result of memory failure, for example), would produce the same result. Thus, although the phenomenology of exaggerated flow is readily observed, by those who run on treadmills, Pelah and Barlow did not develop a convincing way to measure it quantitatively.

Some years ago, in collaboration with Pelah, our lab set out to use a large screen to project an optic flow field and try to have people match the optic flow to their treadmill walking speed before and after adapting to treadmill running. At that time, we had no head-tracking equipment so the visual image was not linked to head movements. We found that people had a very hard time making reasonable matches between their own speed and the visual flow speed. The task did not seem entirely reasonable. This may have been partly due to conflicts between motion parallax and stereo cues, exacerbated the compression of perceived depth in our displays. We abandoned our efforts to measure the flow aftereffect by this method. We did discover that the motion of very slow optic flow rates (corresponding to about a third of walking speed) was completely undetectable while walking on the treadmill which led each of us to become interested in motion suppression phenomena. ${ }^{15-23,25,26}$ We were also interested in adaptation to altered or absent flow. ${ }^{12}$, 27

\subsection{Adapting to altered flow speed}

Rieser et al. provided altered visual flow during locomotion, by pulling a treadmill on a trailer so that visual speed could be made higher or lower than locomotor speed. ${ }^{28}$ Such adaptations affected the calibration of non-visual self-motion perception as measured by visually-directed walking performance. After experiencing slower-than-appropriate visual motion information, participants would overshoot previewed targets that they attempted to walk to without visual feedback. The overshoot is appropriate if the adaptation to reduced flow altered the perception of locomotor action. We have found even stronger effects when adapting people to altered flow in wide area VR while they walked on solid ground and were provided with artificially increased or reduced optic flow. ${ }^{29}$ The recalibration of non-visual selfmotion perception does not require visual feedback, however. It can also be achieved by conflicts between locomotor activity (such as on a treadmill) and non-visually perceived self-motion. ${ }^{12}$ These changes in locomotor calibration can be expressed by other kinds of tasks, such as inadvertent drift when attempting to run, walk or hop in place. ${ }^{27,30-32}$

\subsection{Measuring apparent flow speed}

In studies seeking to determine whether supplying optic flow in treadmill VR would moderate these other kinds of adaptation effects to treadmills we sought again to find flow speeds that seemed subjectively equal to locomotor speeds. We were interested in why people drift forward inadvertently when attempting stationary running with closed eyes after running on a treadmill. ${ }^{30}$ With Pelah, we had shown that removing visual feedback during normal running produced the same "drift" effect. ${ }^{27} \mathrm{We}$ then showed the converse, that adding visual flow during treadmill locomotion reduced the aftereffect. ${ }^{33}$ However, during these investigations, we again noticed that, even in immersive VR, higher visual speeds than appropriate were required to seem "correct".

We thereafter adopted a technique for comparing visual speeds with motor speeds in treadmill VR. Using a method of adjustment, we found that that visual speed was typically set too high by a factor of about 1.5 , but that this factor could be made closer to 1 if gaze were directed downward at the floor or off to the side. ${ }^{26}$ Based on this, it was argued that what is crucially absent in HMDs is the more parallel lines of ("lamellar") optic flow available in the periphery. 
However, it is also possible that this is a matter of absolute retinal flow speeds, ${ }^{19}$ because these were generally faster in the periphery.

Using adaptive methods in treadmill VR, we found that VE speed matches obtained with treadmills were lower when the side walls of the virtual hallway in which locomotion occurred were closer together - producing faster absolute flow rates. ${ }^{34}$ This is similar to the commonly observed differences in subjective speed of self-motion produced by changes in the height of the driver above the road - and also of the effect of painting lines across the road near intersections so as to provide more motion energy, resulting in a higher rate of visually perceived self-motion. In general, the data reported in studies in which the speed of a virtual environment is compared with motor information concerning self-motion is fairly consistent: Visual speeds in HMDs typically need to be set higher than appropriate in order to appear correctly matched to motor activity on a treadmill. But apparent visual speed is increased by making faster retinal flow available by turning the head, enlarging the field of view, or altering the structure of the environment.

\subsection{Gain matching and the flow effect}

Having developed techniques in immersive treadmill VR for measuring apparent matches between visual and kinesthetic estimates of the speed of self-motion ("gain matching"), we have also found that Pelah and Barlow's flow effect can be quantitatively measured by this means. ${ }^{35}$ That is, we can measure visual speed matches before and after adaptation to treadmill locomotion. A flow effect (enhanced visual flow) would be represented by a decrease in matched visual flow. Indeed, when adaptation was to treadmill walking either in a static VE or with eyes closed, we found reductions in visual speed matches in both conditions (by about 10\%) following adaptation. This was somewhat surprising because Pelah and Barlow reported that a greater flow effect is found if adaptation occurs with eyes open. ${ }^{20}$ However, when we used treadmill running for adaptation, we only found evidence for a flow effect (17 percent reduction in visual speed matches) after treadmill running with eyes open. Note that it is possible that the adaptation to treadmill walking, rather than producing enhanced visual flow, was actually producing a reduced sense of kinesthetic speed, because this, too, would result in reduced visual speed matches.

\subsection{Why is gain matching badly calibrated in treadmill VR?}

In general, because gain matching represents a comparison of two quantities (visual flow and biomechanical selfmotion), poor calibration could be due to error in either estimate or bias is their comparison. If kinesthetic speed on a treadmill is overestimated or visual speed is underestimated, the same direction of effect would be found. Conversely, perfect gain matching could arise when both quantities are misperceived by the same amount.

Because we now know there are strong interactions among various cues to self-motion, one prediction of Barlow's intersensory inhibition theory, for example, is that as visual speed increases, estimates of motor speed might decrease. In other words, the perception of either of these two cues involves dynamic interactions between them. It is therefore impossible to completely isolate them from each other, because the manipulation of one cue will affect the other.

In general, a theory of cue inter-calibration would assert that human calibration of self-motion perception ought to be best under conditions that most resemble normal experience. In normal experience, for example, locomotor speed is self-controlled. The lack of control of speed on a treadmill may contribute to an overestimate of locomotor speed. Mittelstaedt and Mittelstaedt found that distance estimates (and presumably speed estimates) deviated from good calibration when subjects were required to walk faster or slower than normal. ${ }^{5}$ These deviations were consistent with exaggerating perceived differences between preferred speed and required speed. Similarly, the apparent speed of walking on a treadmill may be exaggerated by its novelty or by the increased vigilance required to respond to the movement of the treadmill belt.

On the visual side, the perception of speed may or may not entail the perception of motion through space, but we do know that distances are underestimated in VR. While it is possible that visual speeds would be perceived accurately even when visual distances are not, it seems more likely that visual speeds in VR would be similarly underestimated.

\section{EXPERIMENT 1: GAIN MATCHING IN TREADMILL VR AND IN HALLWAY VR}

The purpose of this experiment was to compare gain-matching performance on a treadmill with that on solid ground, and to do so both in a filled and in an empty environment. We wanted to compare filled with empty space, because the 
presence of virtual objects in near space may allow better evaluation of distances and predictions of time to contact. Clutter has been implicated in the perception of heading, for example. ${ }^{36}$ Although the introduction of such clutter has previously shown only small effects on gain-matching in treadmill $\mathrm{VR},{ }^{34}$ it might be that clutter is of more value when an observer also has access to the intercalibrated multisensory estimations of self-motion that are more likely to be available on solid ground than on the less well-calibrated act of treadmill walking., 57,38

We stress that all participants were new to the VR, to avoid contamination from prior experience with the system. Moreover, although both virtual environments were tested within subjects, the two environment used were presented in separate sessions (with a brief break) to avoid inter-environment contamination. These kinds of considerations are of some importance in trying to measure the initial calibration state of our participants. Moreover, the use of sequential sessions in the treadmill VR was fortuitous in revealing an order effect that may be indicative of adaptation to treadmill locomotion.

\subsection{Methods}

\subsubsection{Participants}

Seventeen Swarthmore undergraduates participated for pay or in fulfillment of a course requirement. All were naïve to the hypotheses and had not been in VR before. Eight were assigned to hallway VR and the other nine to treadmill VR.

\subsubsection{Design}

To measure visuo-motor calibration in immersive VR, the point of subjective equality (PSEs) between visual and motor self-motion was assessed using an interleaved staircase method. Two variables were tested. The presence or absence of physical translation was varied between subject by having half do the task in treadmill VR (no physical translation) and half in hallway VR, where they walked on solid ground. The role of environmental structure was tested by having each participant do two sessions, one in which the environmental structure was a textured hallway devoid of objects and the other in which randomly scattered floor-to-ceiling columns were added to the hallway. Environment order was varied between subjects.
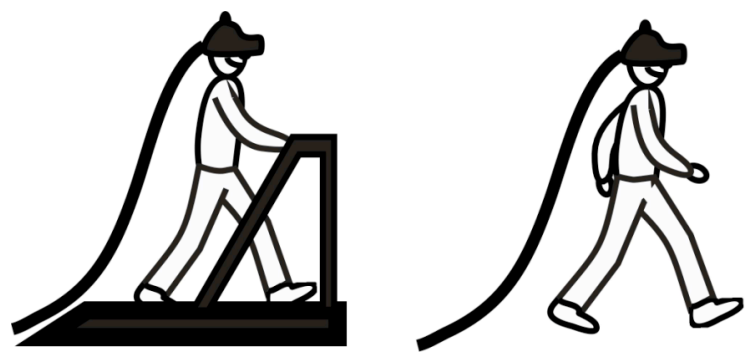

Figure 1. Locomotor activity (walking on a treadmill or walking on solid ground in wide area VR) was varied between subjects.

In each session, 60 trials were conducted based on an adaptive method employing three "staircases" with large stepsizes. One staircase started with a high visual gain (visual speed 1.63 of motor speed), one with a low gain (visual speed 0.61 of motor speed), and one in the middle (gain of 1.0). The extreme values represent 10 steps in the ratio of 1.05 above and below the correct gain. Staircase step size was 3 such ratio steps in response to each trial. Twenty trials from each staircase were randomly ordered. On each trial the observer was required to indicate whether the visual flow was faster or slower than the motor speed. A logit analysis was used to compute PSEs for each observer in each environment.

\subsubsection{Apparatus.}

Our wide area virtual reality set up (Swarthmore College Perception Lab) uses an HMD and an optical tracking system. The HiBall tracker system covered an area $15 \times 2.2 \mathrm{~m}$. The tracker system is fast and accurate, running at over $1000 \mathrm{~Hz}$ with $1 \mathrm{~mm}$ precision. The position signal was averaged over a temporal window of $30 \mathrm{~ms}$ and sampled at $120 \mathrm{~Hz}$ for the present experiments. 
Displays were presented through a V8 stereo LCD HMD with a 60 degree diagonal field of view (38.1 deg vertical; 640 $\mathrm{x} 480$ resolution). The HMD is adjustable and can be worn relatively comfortably for the duration of our experiments. It also has built-in headphones though which sound signals can be given to participants. Hearing-attenuating earplugs (NR 31 ) were used in all conditions to minimize environmental sound localization cues.

Our graphics were rendered at $120 \mathrm{~Hz}$ using OpenGL by 2 Macintosh G4 computers (System 9) with Radeon video cards (one computer for each eye). Graphics were displayed at $60 \mathrm{~Hz}$, using a 2-frame accumulation buffer to approximate motion blur between frames. A third computer controlled the experimental procedure and received input from the experimenter. A fourth computer controlled any sound signals being sent to the headphones on the HMD. The computers communicated via ethernet on a private, dedicated lab network. Based on equipment specifications, we have estimated the lag in our system to be $35 \mathrm{~ms}$ or less. Interpupillary distance was measured with a digital PD meter at the vergence of the V8 $(1 \mathrm{~m})$ and used, along with the HMD vergence angle, in the specification of the stereo graphics.

For treadmill VR, we used a Landice 8700 Sprint treadmill with a belt approximately $45 \mathrm{~cm}$ wide and $130 \mathrm{~cm}$ long.

\subsubsection{Displays}

The two main virtual environments and the motion-neutral environment are shown in Figure 2. The hallways were $2 \mathrm{~m}$ wide and $2.5 \mathrm{~m}$ high and extended to the graphics clipping plane $(100 \mathrm{~m})$. The walls, floor and ceiling of the virtual hallway were covered with a seamless visual texture containing both small and large elements to provide maximal visual information for optic flow at multiple spatial scales. In the columns world, new random column positions were generated for each trial. Each column was $0.2 \mathrm{~m}$ in diameter and textured. The motion-neutral environment allowed participants to orient themselves without providing them with visual feedback about self-motion.
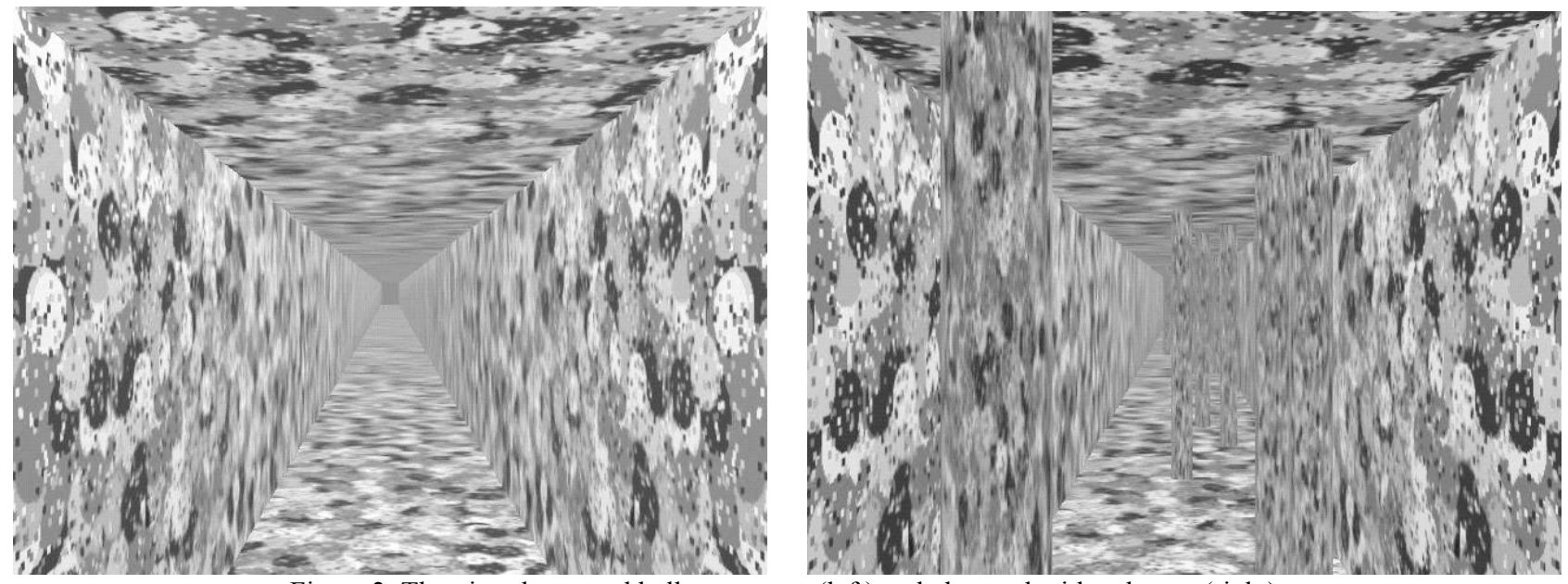

Figure 2. The virtual textured hallway empty (left) and cluttered with columns (right).

\subsubsection{Procedure}

In the treadmill VR, participants would walk continuously on the treadmill at $3 \mathrm{mph}(1.34 \mathrm{~m} / \mathrm{s})$ in the motion-neutral environment. On each trial the display would switch to the appropriate VE with a simulated speed determined by the gain parameter for that trial multiplied by the treadmill speed. After about $4 \mathrm{~s}$ (duration was varied randomly between 3.5 and $4.5 \mathrm{~s}$ ), the display would revert to the motion-neutral environment. The participant would then indicate the speed of the visual world on that trial ("faster" or "slower") relative to their walking speed. A few practice trials were given with extreme gain values ( 2.0 or 0.5$)$ until the participant was comfortable with the task. There were then 60 experimental trials in the appropriate environment. After a short break, a second session of 60 trials was conducted in the other environment (order varied between subjects). Each session took about 10 minutes.

In hallway VR, participants were first required to practice walking at a reasonable speed (at least $1.1 \mathrm{~m} / \mathrm{s}$ ) up and down the hallway before donning the HMD. On each trial, the participant oriented themselves in the positioning hallway (e.g., turned around between trials) and begin walking when the experimental VE was presented. An auditory signal would 
tell them when to stop. If they did not reach the minimum goal speed of $1.1 \mathrm{~m} / \mathrm{s}$ during the trial they were instructed of this and the trial was repeated. In this condition, the setting the visual gain of hallway motion to some value $\mathrm{X}$ meant that for every meter of physical progress along the long axis of the hall, the visual motion of the hall was $\mathrm{X}$ meters. Lateral motion was not distorted (so that motion parallax information from sway was unaffected by the gain manipulation). Note that in this condition, participants accelerated to walking speed in the VE, whereas treadmill VR trials were conducted at constant speed. As in treadmill VR, there were a few initial practice trials, there were 60 experimental trials in each session (one environment per session), and a short break was given between sessions. Each session took about 10 minutes.

\subsection{Results}

PSEs were computed for each subject in each environment based on the 60 experimental trials. Statistical analyses were conducted in $\log$ (ratiometric) space, but the results will be reported in terms of linear ratios between visual and motor speed for clarity.

\subsubsection{Treadmill VR}

As in prior studies, gain matching in treadmill VR resulted in rather high visual speeds. However, there was an unexpected order effect evident in the data. For the first session, the average visual gain at PSE was 1.42, which is similar to previous values. However, the average visual gain for the second session (1.21) was reliably less than that for the first, $\mathrm{t}(8)=2.85, \mathrm{p}<.05$. This order effect swamped any difference between the two environments. Overall, the average gain in the empty hallway (1.32) was no different than that in the hallway with columns $(1.30), t(8)=0.19$, n.s. Numeric differences in the first session alone ( 1.52 for the empty hallway vs. 1.35 for the hallway with columns), were also not reliable, $\mathrm{t}(8)=0.94$, n.s.

\subsubsection{Hallway VR}

In marked contrast to the results from the treadmill VR, gain matching when walking on solid ground showed no evidence of order effects, but was strikingly different for the two VE conditions. When the VE was an empty hallway, gain matches averaged 1.39, which is similar to the value found for treadmill VR. When the VE was populated with columns, however, gain matches in hallway VR averaged 1.06, which was reliably less than those of the empty hallway, $\mathrm{t}(7)=3.67, \mathrm{p}<.05$, but not reliably different from 1.0 .

\subsection{Discussion}

There were two unexpected results from Experiment 1. The first concerned treadmill VR. Although initial gain matches in treadmill VR were quite high, settings in the second session were reliably less, independent of VE. The most likely explanation for this is that our subjects adapted to treadmill walking in the first session, resulting in reduced perceived rate of self-motion in the second session. ${ }^{12}$ For this explanation to make sense, however, seems to require an additional assumption to explain why judgments were stable over the course of the first session (they were). Quite likely, people were not always comparing the visual speed to their motor speed. Rather, because treadmill speed was constant throughout the session, it seems likely that subjects could have developed an internal visual standard for making judgments in the first session, (and referenced that when making judgments). On this view, the change in the second session reflects the need to establish a new internal standard when the visual environment was changed radically by the addition or removal of pillars. On the adaptation account, this second standard was established at a time when the perceived locomotor speed on the treadmill was reduced by adaptation.

More importantly, the second finding of note was evidence of apparently rather good calibration in the more richly structured environment when walking on solid ground. Indeed, given all the evidence concerning poor distance perception in $\mathrm{VR},{ }^{39,40}$ it seems quite surprising that calibration could be as good as this. Of course, it remains possible that the added columns simply raise the absolute rates of optic flow enough to change the apparent matching gain without actually reflecting a truly accurate assessment. However, on this account it seems hard to explain why a similar reduction in gain was not found in treadmill VR for the same rich environment. Before proceeding to interpret this finding further, however, we sought to replicate it with a larger number of participants. 


\section{EXPERIMENT 2: GOOD GAIN MATCHING WITH A RICH VE IN HALLWAY VR}

The apparently excellent gain matching on solid ground in the rich environment differed so markedly from present and previous results with treadmill VR that we decided to replicate the experiment with a larger number of participants. The methods were the same as in the solid-ground condition of Experiment 1 except that only 36 trials were conducted in each session, and there were 13 participants. As before, all participants made relative speed judgments in separate sessions in an empty textured virtual hallway and in a column-filled virtual hallway. Order of session was alternated between subjects.

\subsection{Results}

The results were basically identical to those of the solid-ground condition of Experiment 1. Mean PSEs for the richlystructured environment represented gain ratios of 1.04 whether they were tested first or second. These PSEs did not differ reliably from a gain of $1.0, \mathrm{t}(12)=0.80$, n.s. They did differ reliably from the PSEs in the empty hallway VE (1.29), $\mathrm{F}(1,11)=15.1, \mathrm{p}<.01$. Again, the empty hallway VE gains were the same whether tested first (1.30) or second (1.29). Although there was some variability in the estimates, these results correspond well with those measured in Experiment 1.

\subsection{Combined analysis of solid ground performance}

When the data from all 20 participants who walked on solid ground in the two experiments is analyzed together the following conclusions are supported. First, average gain matches in the hallway cluttered with columns (1.05), did not differ reliably from $1.00, \mathrm{t}(19)=1.19$, n.s. Second, average gain matches in the empty hallway (1.33) were higher than in the columned hallway by a factor of 1.26 . The lower bound on the $5 \%$ confidence interval representing the average difference represents a difference in gain by a factor of 1.15 between the two VEs. This is important, because there is prior evidence that adding columns lowered that matching gain, but only by a factor of $1.09{ }^{34}$. The combined results of all manipulations are summarized in Table 1 .

Table 1. Summary of gain-matching PSEs as a function of walking surface, virtual environment, and order

\begin{tabular}{|ccc|}
\hline & \multicolumn{2}{c|}{ Mean PSE } \\
Treadmill VR (N=9) & Cluttered VE & Empty VE \\
Columns world first & 1.35 & 1.52 \\
Empty hall first & 1.25 & 1.19 \\
Overall & $1.30 \pm 0.09$ & $1.32 \pm 0.10$ \\
& & \\
Solid ground VR (N=20) & 1.04 & 1.29 \\
Columns world first & 1.04 & 1.30 \\
Empty hall first & $1.05 \pm .05$ & $1.29 \pm 0.08$ \\
Overall & & \\
\hline
\end{tabular}

\subsection{Discussion}

The results of the present experiment confirm that the matching of visual and non-visual speeds of self-motion can approach accuracy when walking on solid ground in a VE that includes near objects. Average gains at PSE in this condition departed numerically from 1.0 by only about $5 \%$, and did not depart statistically from 1.0. Durgin and Kearns originally reported similar performance in treadmill VR, but later determined that their gain calculations had been in error by a factor of 1.41 because of a programming error. In fact, using somewhat different procedures, they found that adding columns to the empty textured hallway lowered gain matches by a factor of about 1.09. Similarly, in Experiment 1, between subject analyses of the first block of trials, suggest that gains are higher without columns by a ratio of 1.07. We now consider some possible explanations of the excellent performance on solid ground in a cluttered VE.

\subsubsection{Refutation of the increased retinal flow explanation}

Durgin and Kearns showed that dramatic changes in apparent world speed (and in gain matches in treadmill VR) could be induced by narrowing or widening the virtual hallway. ${ }^{34}$ The introduction of columns in the present experiment however, produced only a small (and unreliable) change in gain matches in the treadmill VR. Thus, the improved performance in the richer environment when walking on solid ground does not seem to be attributable primarily to 
higher rates of retinal flow produced by nearer surfaces because the amount of change is far greater than found with the same visual change in treadmill VR.

\subsubsection{Refutation of the slow walking speed explanation}

Banton et al. argued that gain matching was good when treadmill speeds were slower than normal. ${ }^{26}$ Is it possible that the acceleration period allowed the self-motion systems to sweep through this supposedly better-calibrated region of motor speeds? This kind of account could clearly predict no difference, however, between the two VEs. Moreover, evidence from open-loop walking suggests that although people are well-calibrated at their normal walking speed even without training, they overestimate the extent of speed change when they depart from normal walking speeds. ${ }^{5}$ Thus, the report of Banton et al. of more accurate (lower) visual speed settings at low locomotor speeds probably reflects an underestimation of walking speed offsetting the underestimation of visual speed. It is a property of the gain-matching task that it does not provide an independent assessment of the calibration of either system. Independent evidence from motor tasks suggests, however, that the motor system is best calibrated at normal walking speeds, not slow ones.

\subsubsection{Consideration of the effects of distance compression in VR}

One straightforward explanation of the failure of gain matching in general is the misperception of distance in VR. ${ }^{39}, 40$ Indeed, in visually-directed walking tasks performed in the empty hallway VE in our lab, we find that people walk only about $70 \%$ of the rendered distance, whereas in the real hallway people tend to walk $95 \%$ of previewed distances without any training. ${ }^{29}$ Such discrepancy suggest that distances in the empty hallway VE are compressed by a factor of approximately 0.74 . Such distance compression, if converted to visual speed perception, should result in gains of approximately 1.36 , which corresponds well with the gain matches obtained in the empty hallway VE when our participants walked on solid ground. Because we have not directly assessed distance perception in a cluttered VE, it would be interesting to learn whether it differs in any way, though our subjective impressions do not suggest it would.

It remains possible that near-space distances are better calibrated in our VEs and that only in the cluttered environment can ones motion be judged with respect to object that pass into near space $(<1 \mathrm{~m})$.

\subsubsection{The possible role of time-to-contact information}

We have recently begun investigating time-to-contact judgments while standing or walking in VR. Preliminary result suggest that judgments of time to contact depend not only on retinal analyses of object expansion, but also on estimates of object velocity (e.g., from stereo). During walking TTC estimates, for identical displays, were earlier than when standing. These preliminary observations suggest that walking may decrease time to contact estimates. This could have played a role in the cluttered environment.

\subsubsection{Why is performance on solid ground so different from treadmill VR?}

Analysis of head motions during treadmill and solid ground walking suggest that they are similar with respect to the amplitude of bob (vertical translation of the head with each stride) and sway (horizontal translation of the head with each stride). Lunge (evidenced by periodic accelerations and decelerations in the direction of travel) is sometimes present on solid ground and sometimes not. It seems somewhat less frequent on treadmills, though we have not made a detailed study of this. Thus, we do not think that the periodic vestibular signals are very different in the two circumstances.

Similarly, although walking on solid ground is known to be well calibrated, the role of biomechanical or kinesthetics is probably more important than the role of inertial or vestibular signals of overall velocity. ${ }^{5}$ We find that when accelerations profiles are varied, estimates of velocity of passive self motion are as strongly influenced by peak acceleration as by peak velocity. In normal walking, peak acceleration during the first step is probably tightly correlated with peak velocity, so normal calibration may take advantage of this. ${ }^{8}$

One very important difference between treadmill locomotion and that on solid ground has to do with the control structures involved. In the case of our treadmill VR, the real objective of motor activity is to avoid falling from the treadmill. That is, motor parameters are tuned to maintain a stationary position relative to the treadmill by means of haptic contact. ${ }^{12}$ In contrast, walking through the hallway VR includes real guidance of motor steering (participants 
know that they will not bump into anything as long as they stay within the walls of the hallway). Although this difference is subtle, it may be of crucial importance insofar as obstacle avoidance systems might be most sensitive to discrepancies from normal calibration, but only if they are engaged by the motor demands of the task - and only if they receive relevant information (obstacles!) which are only available in the cluttered environment.

Investigations of gain matching during passive self-motion have proven difficult. When we ask people to assess relative speed of optic flow (presented in an HMD) and passive self-motion, we find that, although they can do the task, their

perceptions are fairly variable and seem to drift (adapt) in a manner suggestive of visual capture. ${ }^{14}$ That is, each test trial may re-center the system to some degree and contaminate the very thing one is trying to measure. By using a method of constant stimuli, however, we find that discrimination thresholds in this task are on the order of magnitude of $15 \%$.

Similarly, in recent work measuring discrimination thresholds for peak speed of passive self-motion (without visual feedback), we find that discrimnation thresholds are really quite high (Weber fractions of $15 \%$ ), whereas discriminations of peak accelerations are more impressive (about 5\%). ${ }^{8}$ The opposite patterns of sensitivity emerge for judgments of optic flow. Speed discriminations are excellent (3\%), while discriminations of acceleration are poor. The high discrimination thresholds for comparing passive self-motion and visual flow (even in a cluttered environment) as well as the visual capture of vestibular speed, both make sense based on these kinds of differential sensitivities.

\section{SUMMARY AND CONCLUSIONS}

We have used a task where people are asked to compare visual with biomechanical self-motion information. The ecological validity of the task is suggested by the observation that, after treadmill running, people readily notice a discrepancy between expected and perceived visual flow when walking. ${ }^{20}$ Prior "gain-matching" studies using treadmill VR have reported that people tend to set the visual speed too high. ${ }^{26}$ Here we have replicated this mis-calibration for treadmill VR using both empty (though well-textured) and cluttered environments. However, we have also found that gain matches are quite close to accurate when walking on solid ground in a wide-area VR system displaying a cluttered environment. Insofar as treadmill locomotion differs from normal walking, it may be argued that the inter-calibration of visual and non-visual sources of information is best when action and vision most resemble those calibrated in normal perception.

It must be emphasized that the intended implication of these findings does not concern the calibration of visual selfmotion perception, per se. We now know that visual flow fields normally appear slower during self-motion than the same fields appear when stationary. ${ }^{19}$ The "correct" cognitive estimation of flow rate is probably irrelevant to behavior, however (much as veridical cognitive estimations of geographical variables like slant appear to be inessential ${ }^{42}$ ). What matters is the complex coordination and inter-calibration of many different sources of visual information. The reduction of perceived visual flow during self-motion is accompanied by enhanced discrimination among visual speeds in the expected range. ${ }^{18}$ This is the prediction of Barlow's theory of inter-dimensional normalization. ${ }^{24}$, 43 Appropriately, discrimination is more important than direct estimation in the case of visual flow rates. Biomechanical variables (intercalibrated with other sensory sources) may predominate in estimating self-motion, ${ }^{5}$ while optic flow and other kinds of self-motion information are used to continuously tune the system. ${ }^{12}{ }^{28}$ For obstacle avoidance, however, accurate mappings between perception and action are quite important. Although further experiments are needed to isolate the critical variables responsible for good inter-calibration, the present data are consistent with the idea that when normal near-space navigation systems are engaged, inter-modal discrepancies are readily detected: Real walking among virtual objects that enter near space demonstrates very good inter-calibration between action and visual perception in wide area VR.

\section{ACKNOWLEDGMENTS}

This work was supported by HHMI and by a Swarthmore College Faculty Research Grant. 


\section{REFERENCES}

1. J. M. H. Beusmans, "Optic flow and the metric of the visual ground plane," Vision Research 38, pp. 1153-1170, 1998.

2. M. Lappe, F. Bremmer, and A. van der Berg, "Perception of self-motion from visual flow," Trends in Cognitive Science 9, pp. 329-336, 1999.

3. F. Redlick, M. Jenkin, and L. Harris, "Humans can use optic flow to estimate distance of travel," Vision Research 41, pp. 213-219, 2001.

4. H. Frenz, F. Bremmer, and M. Lappe, "Discrimination of travel distances from 'situated' optic flow," Vision Research 43, pp. 2173-2183, 2003.

5. M.-L.Mittelstaedt, and H. Mittelstaedt, "Idiothetic navigation in humans: Estimations of path length," Experimental Brain Research 139, pp. 318-332, 2001.

6. I. Israël, and A. Berthoz, "Contribution of the otoliths to the calculation of linear displacement," Journal of Neurophysiology 62, pp. 247-263, 1989.

7. L. R. Harris, M. Jenkin, and D. C. Zikovitz, "Visual and non-visual cues in the perception of linear self-motion," Experimental Brain Research 135, pp. 12-21, 2000.

8. E. Schaffer, and F. H. Durgin, "Visual-vestibular dissociation: Differential sensitivity to acceleration and velocity," submitted.

9. J. Dichgans, and T. Brandt, "Visual-vestibular interaction: Effects on self-motion perception and postural control," in Handbook of Sensory Physiology, Volume 8: Perception, R. Held, H. W. Leibowitz, and H.-L. Teuber eds., pp 755-804, Springer Verlag, Berlin, 1978

10. T. Mergner, and T. Rosemeier, "Interaction of vestibular, somatosensory and visual signals for postural control and motion perception under terrestrial and microgravity conditions: A conceptual model," Brain Research Reviews 28, pp. 118-135, 1998.

11. T. Brandt, P. Bartenstein, A. Janek, and M. Dietrich, "Reciprocal inhibitory visual-vestibular interaction: Visual motion stimulation deactivates the parieto-insular vestibular cortex," Brain 121, pp. 1749-1758, 1998.

12. F. H. Durgin, A. Pelah, L. F. Fox, J. Lewis, R. Kane, and K. A. Walley, "Self-motion perception during locomotor recalibration: More than meets the eye," Journal of Experimental Psychology: Human Perception and Performance, in press.

13. J. J. Gibson, The senses considered as perceptual systems, Boston, Houghton-Mifflin, 1966.

14. J. R. Lishman, and D. N. Lee, "The autonomy of visual kinaesthesis," Perception 2, pp. 287-294, 1973.

15. H. Wallach, and E. W. Flaherty, "A compensation for field expansion caused by moving forward," Perception \& Psychophysics 17, pp. 445-449, 1975.

16. B. Pavard, and A. Berthoz, "Linear acceleration modifies the perceived velocity of a moving visual scene," Perception 6, pp. 529-540, 1977.

17. L. R. Harris, M. J. Morgan, and A. W. Still, " Moving and the motion after-effect," Nature 293, 139-141, 1981.

18. F. H. Durgin, K. Gigone, and E. Schaffer, "Improved visual speed discrimination while walking," [abstract] Journal of Vision, 2004.

19. F. H. Durgin, K. Gigone, and R. Scott, "The perception of visual speed while moving," Journal of Experimental Psychology: Human Perception and Performance, in press.

20. A. Pelah, A. and H. B. Barlow, "Visual illusion from running," Nature 381, p. 283, 1996.

21. A. E. I. Thurrell, A. Pelah, and H. K. Distler, "The influence of non-visual signals of walking on the perceived speed of optic flow," [Abstract]. Perception, 27, 147-148, 1998.

22. A. Pelah, and A. E. I. Thurrell, "Reduction of perceived visual speed during locomotion: Evidence for quadrupedal perceptual pathways in human?" [Abstract]. Journal of Vision 1, p. 307a, 2001.

23. H. K. Distler, A. Pelah, A. G. Bell, and A. E. I. Thurrell, "The perception of absolute speed during self-motion," Poster presented at the annual meeting of the European Conference on Visual Perception, Oxford, England, 1998.

24. H. B. Barlow, "A theory about the functional role and synaptic mechanism of visual aftereffects," in Vision: Coding and Efficiency, C. Blakemore, ed., pp. 363-375, Cambridge, England: Cambridge University Press, 1990.

25. A. Pelah, and A. Boddy, "Adaptive modulation of the motion after-effect by walking," [Abstract] Journal of Physiology-London 506P, 111P-112P, 1998. 
26. T. Banton, J. Stefanucci, F. H. Durgin, A. Fass, and D. Proffitt, "Perception of walking speed in virtual environments," Presence: Teleoperators and Virtual Environments, in press.

27. F. H. Durgin, and A. Pelah, "Visuomotor adaptation without vision?," Experimental Brain Research 127, pp. 12-18, 1999.

28. J. J. Rieser, H. L. Pick, Jr., D. Ashmead, and A. Garing, "Calibration of human locomotion and models of perceptual-motor organization," Journal of Experimental Psychology: Human Perception and Performance 21, pp. 480-497, 1995.

29. F. H. Durgin, L. F. Fox, J. Lewis, and K. A. Walley, "Perceptuomotor adaptation: More than meets the eye," Abstracts of the Psychonomic Society 7, pp. 103-104, 2002.

30. S. M. Anstis, "Aftereffects from jogging," Experimental Brain Research 103, pp. 476-478, 1995.

31. D. R. Proffitt, J. Stefanucci, T. Banton, and W. Epstein, "The role of effort in perceiving distance", Psychological Science 14, pp. 106-112, 2003.

32. F. H. Durgin, L. F. Fox, and D. H. Kim, "Not letting the left leg know what the right leg is doing: Limb-specific locomotor adaptation to sensory-cue conflict," Psychological Science 16, pp 576-572, 2003.

33. F. H. Durgin, T. Banton, K. Walley, D. R. Proffitt, J. Steve, and J. Lewis, "Perceptuomotor recalibration in a virtual world," [abstract] Investigative Ophthalmology \& Visual Science 41, p. S799, 2000.

34. F. H. Durgin, and M. J. Kearns, "The calibration of optic flow produced by walking: The environment matters," [abstract] Journal of Vision 2, p 429a, 2002.

35. L. F. Fox, and F. H. Durgin, "Visual illusion from walking" Presented at the second annual meeting of the Vision Sciences Society, Sarasota FL, May, 2001.

36. J. E. Cutting, and P. M. Vishton, "Perceiving layout and knowing distances: The integration, relative potency, and contextual use of different information about depth," in Handbook of perception and cognition (2nd ed.):

Perception of space and motion, S. J. Rogers, and W. Epstein, eds., pp. 69-117, San Diego, CA, Academic Press, 1995.

37. J. J. Rieser, D. H. Ashmead, C. R. Talor, and G. A. Youngquist, "Visual perception and the guidance of locomotion without vision to previously seen targets," Perception 19, pp. 675-689, 1990.

38. J. M. Loomis, J. A. Da Silva, N. Fujita, and S. S. Fukusima, "Visual space perception and visually directed action," Journal of Experimental Psychology: Human Perception and Performance 18, pp. 906-921, 1992.

39. J. M. Loomis and J. M. Knapp, "Visual perception of egocentric distance in real and virtual environments," in Virtual and Adaptive Environments, L. Hettinger and M. Haas, eds., pp. 21-46, Erlbaum, Hillsdale, NJ, 2003.

40. W. B. Thompson, P. Willemsen, A. Gooch, S. H. Creem-Regehr, J. Loomis, and A. Beall, "Does the quality of the computer graphics matter when judging distances in visually immersive environments?," Presence: Teleoperators and Virtual Environments 13, pp. 560-571, 2004.

41. P. Thibodeau, D. Gromko, and F H. Durgin, "Walking and the role of speed in the perception of time to contact," submitted.

42. D. R. Proffitt, M. Bhalla, R. Gossweiler, and J. Midgett, "Perceiving geographical slant," Psychonomic Bulletin \& Review 2, pp. 409-428, 1995.

43. C. W. G. Clifford, and P. Wenderoth, "Adaptation to temporal modulation can enhance differential speed sensitivity," Vision Research 39, pp. 4324-4332, 1999. 